\title{
Mononucleosis Therapy
}

Infectious mononucleosis is a common, world-wide distributed disease, with a broad spectrum of clinical presentations ranging from inapparent infection to a fatal illness (1). One of the clinical presentations of infectious mononucleosis is the anginose type, characterized by sore throat, dysphagia and enlarged tonsils covered by a pseudo-membranous exudate resembling diphtheria. Additional findings include high fever, prostration, marked cervical lymphadenopathy and hepatomegaly.

At present no drug has been widely accepted as a useful therapeutic modality for the anginose type of infectious mononucleosis. Steroids, metronidazole, acyclovir, interferon, cephalexin and cimetidine have all been proposed (2-8). Corticosteroids, previously indicated only in cases of acute upper airway obstruction, have been recently used and are being advocated in "regular" cases of infectious mononucleosis in adults and it seems to be agreed that they shorten the duration of fever and reduce the severity of the symptoms associated with the disease.

Following some favorable reports by Hedstrom et al. and Davidson et al. (4-6) we began to treat children with the anginose type of infectious mononucleosis with metronidazole (Flagyl ${ }^{\circledR}$ ) in a dose of $25 \mathrm{mg} / \mathrm{kg} /$ day t.i.d. for five to seven days. To date we have treated more than 70 children in the age group 1 to 14 years (most being 2 to 9 years of age).

We have observed a striking response to the drug in the vast majority of cases with rapid defervescence within 24 to 36 hours after initiation of therapy. The fever drops per crisis (while in untreated cases it gradually falls by lysis over a period of several days). Concomitantly, there is a rapid improvement in the patients' general condition and within an additional two days there is a complete clearing of the pseudomembranes. No "rebound" phenomena have been noted when the drug was discontinued. No adverse clinical or laboratory effects have been encountered and the serological response to Epstein-Barr Virus was not altered.

The mechanism of the clinical response exerted by metronidazole is not clear. It has been postulated that anaerobic bacteria are associated with the tonsillitis of infectious mononucleosis, therefore metronidazole, being highly effective against anaerobic bacteria, acts simply by curing the "anaerobic tonsillitis". Although this possibility cannot be excluded in our cases, our experience with clindamycin therapy in 12 cases with the anginose type of infectious mononucleosis was disappointing. Clindamycin had no influence on the clinical course and in most cases the drug was discontinued and substituted by metronidazole. Our experience is not based on a double-blind controlled study. Nevertheless it is our strong impression that metronidazole is an excellent drug for the anginose type of infectious mononucleosis. We do not advocate this treatment for the severe cases of upper airway obstruction where steroids are preferable and at times, life saving. However, in the less severe cases metronidazole is by far the better drug since it acts rapidly and does not carry the hazards of immunosuppression exerted by steroids in this potentially oncogenic disease.

It is surprising that this therapeutic modality has not been mentioned thus far in American literature. In Scandinavian literature there are only two controlled studies which have yielded conflicting results $(5,9)$. Hence additional double-blind clinical trials are needed to provide more definitive data. However, our experience lead us to conclude that metronidazole may be a valuable addition to the current therapy of infectious mononucleosis.

\section{Zvi Spirer, Gabriel J. Hauser, Itamar Shalit}

Prof. Z. Spirer, M.D., G. J. Hauser, M.D., I. Shalit, M.D., Departments of Pediatrics and Infectious Diseases, Tel-Aviv Medical Center, Rokach Hospital, Tel Aviv 61000, Israel.

\section{Literature}

1. Schooley, R. T., Dolin, R.: Epstein-Barr Virus (infectious mononucleosis). In: Mandell, G. L., Douglas, R. G., Bennett, G. E. (eds.): Principals and practice of infectious diseases. John Wiley \& Sons, New York 1985, p. 971.

2. Bender, C. E.: The value of corticosteroids in the treatment of infectious mononucleosis, JAMA 199 (1967) 97.

3. Grose, C.: The many faces of infectious mononucleosis: the spectrum of Epstein-Barr Virus infection in children. Pediatrics in Review 7 (1985) 35-44.

4. Hedstrom, S. A., Mårdh, P. E., Ripa, T.: Treatment of anginose infectious mononucleosis with metronidazole. Scand. J. Infect. Dis. 10 (1978) 7-9.

5. Hedstrom, S. A.: Treatment of anginose infectious mononucleosis with metronidazole. A controlled clinical and laboratory study. Scand. J. Infect. Dis. 12 (1980) 265-269.

6. Davidson, S., Kaplinsky, C., Frend, M., Rotem, J.: Treatment of infectious mononucleosis with metronidazole in the pediatric age group. Scand. J. Infect. Dis. 14 (1982) 103-104.

7. Lakie, J.: Use of cephalexin to treat glandular fever: pilot study. Br. Med. J. 286 (1983) 1617-1620.

8. Sacher, R. A., Temple, M. J., Kelly, A. P.: Cimetidine and mononucleosis. Ann. Int. Med. 99 (1985) 410-411.

9. Spelman, D. W., Newton-John, H. F*: Metronidazole in the treatment of anginose infectious mononucleosis. Scand. J. Infect. Dis. 14 (1982) 99-101. 